\title{
Zur Kenntnis der 1-Methylisatine
}

\author{
von

\begin{abstract}
Moritz Kohn und Alfons Ostersetzer.
\end{abstract} \\ (Vorgelegt in der Sitzung vom 13. Februar 1913.)
}

Durch $\mathrm{Helle} \mathrm{r}^{1}$ ist gezeigt worden, daß Isatin bei der Einwirkung von Natriumäthylat das N-Isatinnatrium liefert, welches bei zweistündigem Erhitzen mit der vierfachen Menge Jodmethyl auf $100^{\circ}$ sich zum 1-Methylisatin umsetzt. Man kann jedoch die Darstellung des N-Isatinnatriums in reiner Form, die Anwendung des Jodmethyls und das Arbeiten in geschlossenen Röhren umgehen, wenn man Isatin $(1 \mathrm{Mol})$ mit 25 prozentiger, methylalkoholischer Kalilauge (1 Mol) zusammenbringt und den entstandenen dunklen Brei des N-Kaliumsalzes (I) mit Dimethylsulfat übergießt. Unter stürmischer Reaktion entsteht hierbei das 1-Methylisatin (II).

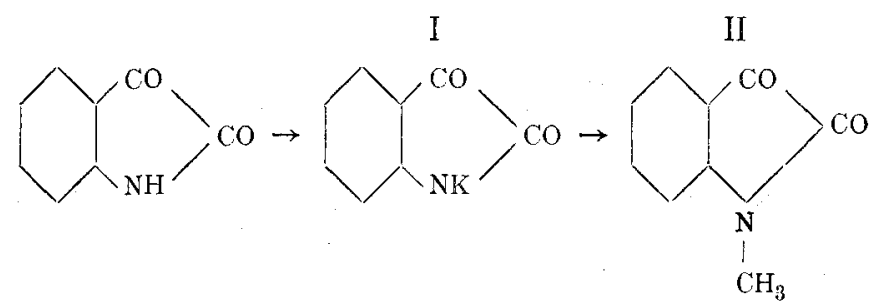

Wir haben in der analogen Weise aus 5-Bromisatin (III) das 1-Methyl-5-Bromisatin (IV)

1 Berl. Ber., 40, 1295 (1907). 


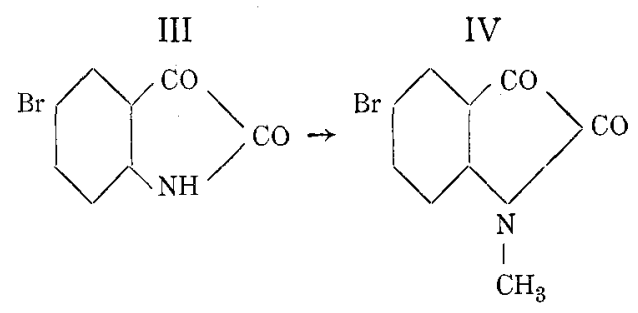

und aus 5,7-Dibromisatin (V) das 1-Methyl-5, 7-Dibromisatin(VI) gewonnen.

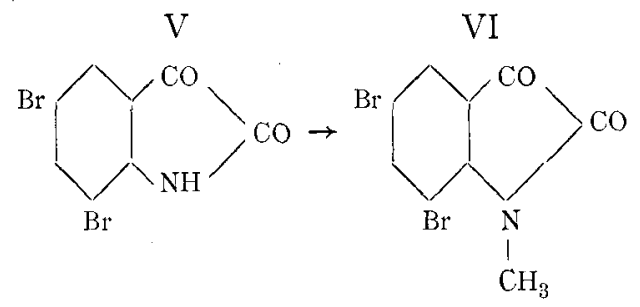

Das 1-Methylisatin liefert bei der Einwirkung von Bromwasser in wässeriger Lösung ein Bromprodukt, welches sich als identisch erweist mit dem 1-Methyl-5-Bromisatin (IV).

Als diese Versuche abgeschlossen waren erschien eine Publikation von Friedländer und Kielbasinski, ${ }^{1}$ in der die Darstellung des N-Methylisatins durch Einwirkung von Dimethylsulfat auf eine Lösung von Isatin in wässerigem Alkali beschrieben wird.

Bei der reduzierenden Acetylierung des 1-Methylisatins erhält man einen schön krystallisierten Körper. Die Analyse, Acetylbestimmung und Molekulargewichtsbestimmung lehrte, daß das Diacetyl 1,1'-Dimethylisatyd (VII)

vorliegt. ${ }^{2}$

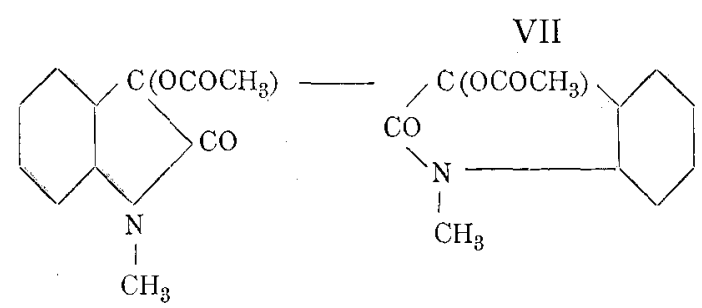

1 Berl. Ber., 44, 3098 u. f. (1911).

2 Die Entstehung des Diacetyl-1, 1'-Dimethylisatyds bietet nicht nur eine wertvolle Bestätigung der seinerzeit über die reduzierende Acetylierung des 
Bei der Einwirkung von Phenylmagnesiumbromid auf das 1-Methylisatin entsteht ein Körper $\mathrm{C}_{21} \mathrm{H}_{17} \mathrm{ON}$. Seine Bildung ist wohl derart zu erklären, daß beide Carbonylgruppen des Methylisatins mit dem Grignard'schen Reagens zur Umsetzung gelangt sind, wobei der ditertiäre Alkohol (VIII) entstehen müßte, dessen Anhydrisierungsprodukt der Körper $\mathrm{C}_{21} \mathrm{H}_{17} \mathrm{ON}$ (IX) wäre.<smiles>CN1C(=O)OC2CCCCC21</smiles><smiles>CCCCCC(O)(CCCCC)C1CCCCC1N(C)c1ccccc1</smiles><smiles>[Y1]C12OC(CCC)(CCC)C1[Y](C)C1CCCCC12</smiles>

1-Methylisatin (N-Methylisatin) (II).

Isatin (1 Mol) wird in einem weithalsigen Kolben mit der berechneten Menge 25 prozentiger methylalkoholischer Kalilauge (1 Mol $\mathrm{KOH}$ ) übergossen. Es bildet sich sofort das blaue Kaliumsalz. Man rührt die breiige Masse rasch mit einem starken Glasstab um, um eventuell unangegriffenes Isatin mit der Lauge in Berührung zu bringen, und verbindet den Kolben rasch mit einem Rückflußkühler. Man läßt durch das Kühlrohr etwas mehr als $1 \mathrm{Mol}$ Dimethylsulfat zufließen. In wenigen Augenblicken setzt eine stürmische Reaktion ein; die Masse erhitzt sich bis zum Sieden. Bei der Verarbeitung größerer Quantitäten muß durch zeitweises Einstellen des Kolbens in kaltes Wasser und sukzessiven Zusatz des Dimethylsulfats die

Isatins sowie des 5-Bromisatins mitgeteilten Beobachtungen, sondern sie erscheint auch deshalb bemerkenswert, weil hier die Stammsubstanz, das 1, 1'-Dimethylisatyd

unbekannt ist.

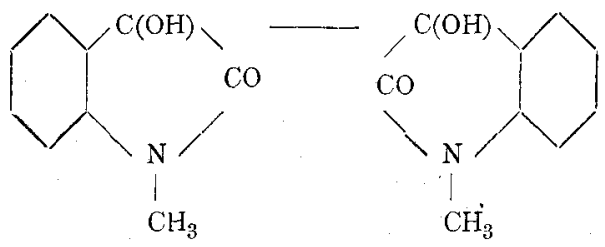


Reaktion gemäßigt werden. Nachdem das Gemisch sich beruhigt hat, wird es im siedenden Wasserbad einige Minuten erwärmt, um die Reaktion zu vervollständigen. Män gießt sodann. den Kolbeninhalt in das mehrfache Volum kaiten Wassers ein und versetzt mit Kalilauge, bis alles in Lösung gegangen ist. Nach dem Ansäuern mit Salzsäure (1:1) krystallisiert das Methylisatin in roten Nädelchen aus, welche abgesaugt und durch Umkrystallisieren aus heißem Wasser gereinigt werden. Es schmilzt bei $132^{\circ}$ (Heller, a. a. O. $134^{\circ}$ ).

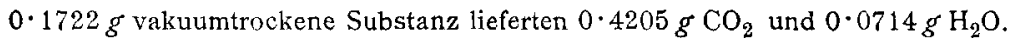

In 100 Teilen:

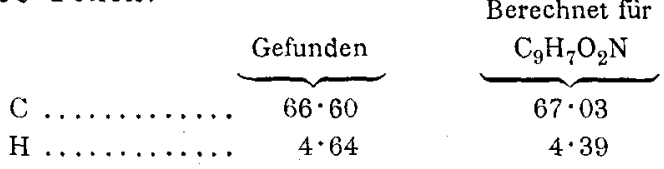

Das reine Methylisatin muß sich in Kalilauge mit rein gelber Farbe ohne vorübergehende Violettfärbung (Verunreinigung mit Isatin) lösen.

\section{1-Methyl-5-Bromisatin (IV).}

Die Darstellung wird in der analogen Weise aus 5-Bromisatin vorgenommen. Die Umsetzung des Kaliumsalzes mit dem Dimethylsulfat verläuft hier weniger stürmisch wie beim Isatin. Das aus der alkalischen Lösung durch Ansäuern ausgefällte Rohprodukt wird abgesaugt und durch Umkrystallisieren aus verdünntem Weingeist gereinigt. Man erhält so feine rote Nadeln, die um $155^{\circ} \mathrm{zu}$ erweichen beginnen und unscharf bei $164^{\circ}$ schmelzen.

I. $0 \cdot 1674 \mathrm{~g}$ vakuumtrockene Substanz lieferten $0.2738 g \mathrm{CO}_{2}$ und $0: 0414 \mathrm{~g}$ $\mathrm{H}_{2} \mathrm{O}$.

11. $0.2122 g$ Substanz lieferten $0 \cdot 1659 g \mathrm{AgBr}$.

In 100 Teilen:

\begin{tabular}{|c|c|c|c|}
\hline & \multicolumn{2}{|c|}{ Gefunden } & \multirow{2}{*}{$\begin{array}{l}\text { Berechnet für } \\
\mathrm{C}_{9} \mathrm{H}_{6} \mathrm{O}_{2} \mathrm{NBr}\end{array}$} \\
\hline & $I$ & II & \\
\hline$C \ldots$. & $44 \cdot 61$ & - & $44 \cdot 99$ \\
\hline$H \ldots \ldots$ & $2 \cdot 76$ & - & $2 \cdot 52$ \\
\hline $\mathrm{Br} \ldots \ldots$ & - & $33 \cdot 27$ & $33 \cdot 31$ \\
\hline
\end{tabular}


Das gleiche Bromprodukt bildet sich beim Versetzen wässeriger Lösungen des Methylisatins mit Bromwasser. Die Fällung wird abgesaugt und aus wässerigem Weingeist umkrystallisiert. Man erhält so rote Nadeln, welche bei etwa $155^{\circ}$ erweichen und unscharf bei $164^{\circ}$ schmelzen. Ein fein verriebenes Gemenge des durch Methylierung des Bromisatins gewonnenen Präparates mit dem Bromprodukt des Methylisatins schmolz ohne eine Spur einer Depression.

\section{1-Methyl-5, 7-Dibromisatin (VI).}

Die Darstellung ist analog der Darstellung des 1-Methyl5-Bromisatins. Die Umsetzung der Kaliumverbindung mit dem Dimethylsulfat verläuft hier noch träger. Man muß daher im Wasserbad erwärmen. Das aus der alkalischen Lösung durch Ansäuern ausgefällte und sodann abgesaugte Rohprodukt wurde durch Umkrystallisieren aus wässerigem Alkohol als sandiges Krystallmehl von ziegelroter Farbe erhalten. Unter dem Mikroskop sieht man nadelige Kryställchen.

I. $0 \cdot 2213 g$ Substanz lieferten $0 \cdot 2720 g \mathrm{CO}_{2}$ und $0 \cdot 0332 g \mathrm{H}_{2} \mathrm{O}$.

II. $0 \cdot 2474 \mathrm{~g}$ vakuumtrockene Substanz lieferten $0 \cdot 2934 \mathrm{~g} \mathrm{Ag} \mathrm{Br}$.

In 100 Teilen:

\begin{tabular}{|c|c|c|c|}
\hline \multirow[t]{2}{*}{$\sqrt{2}+x^{2}$} & \multicolumn{2}{|c|}{ Gefunden } & \multirow{2}{*}{$\begin{array}{r}\begin{array}{r}\text { Berechnet für } \\
\mathrm{C}_{9} \mathrm{H}_{5} \mathrm{O}_{2} \mathrm{NBr}_{2}\end{array} \\
\end{array}$} \\
\hline & I & II & \\
\hline$c \ldots . .$. & $33 \cdot 52$ & - & $33 \cdot 85$ \\
\hline $\mathrm{H} \ldots \ldots \ldots$ & $1 \cdot 67$ & - & $1 \cdot 58$ \\
\hline Br....... & - & $50 \cdot 56$ & $50 \cdot 13$ \\
\hline
\end{tabular}

Der Schmelzpunkt ist $171^{\circ}$ (unscharf).

\section{Diacetyl-1, 1'-Dimethylisatyd (VII).}

Vakuumtrockenes Methylisatin wird mit frisch destilliertem, reinstem Essigsäureanhydrid, dem man einige Tropfen Eisessig zugesetzt hat, unter Rückfluß gekocht und in die siedende Flüssigkeit Zinkstaub in kleinen Anteilen eingetragen, bis Entfärbung eingetreten ist. Man filtriert siedendheiß durch ein gehärtetes Filter den überschüssigen Zinkstaub ab und versetzt 
das Filtrat mit dem mehrfachen Volum Wasser. Man rührt um und läßt mehrere Stunden stehen, bis das Essigsäureanhydrid zersetzt ist.

Das krystallinische Rohprodukt wird abgesaugt und durch Umkrystallisieren aus heißem Alkohol, in dem die Substanz sich in reichlicher Menge löst, gereinigt. Durch neuerliches Umlösen in kochendem Alkohol erhält man rein weiße, glänzende, rhomboedrische Krystalle, die deutlich nach den Flächen spaltbar sind. Die Substanz bräunt sich von etwa $200^{\circ}$ an und schmilzt zwischen 218 und $220^{\circ}$.

I. $0.1977 \mathrm{~g}$ Substanz lieferten $0.4725 \mathrm{~g} \mathrm{CO}_{2}$ und $0.0951 \mathrm{~g} \mathrm{H}_{2} \mathrm{O}$.

II. $0 \cdot 1997 g$ Substanz lieferten $0 \cdot 4772 g \mathrm{CO}_{2}$ und $0.0952 g \mathrm{H}_{2} \mathrm{O}$.

In 100 Teilen:

\begin{tabular}{|c|c|c|c|}
\hline & \multicolumn{2}{|c|}{ Gefunden } & \multirow{2}{*}{$\begin{array}{r}\begin{array}{r}\text { Berechnet für } \\
\mathrm{C}_{22} \mathrm{H}_{20} \mathrm{O}_{6} \mathrm{~N}_{2}\end{array} \\
\end{array}$} \\
\hline & I & II & \\
\hline $\mathrm{C}$ & $65 \cdot 18$ & $65 \cdot 17$ & $64 \cdot 67$ \\
\hline$\ldots$. & $5 \cdot 38$ & $5 \cdot 33$ & $4 \cdot 95$ \\
\hline
\end{tabular}

Die Molekulargewichtsbestimmung nach der ebullioskopischen Methode ergab:

$0.4274 g$ erhöhten den Siedepunkt von $21.82 g$ Benzol um $0 \cdot 115^{\circ}$.

Daraus berechnetes Molekulargewicht:

$m \ldots \ldots \ldots \ldots, 442 \cdot 8 \quad \underbrace{\begin{array}{c}\text { Berechnet für } \\ \mathrm{C}_{22} \mathrm{H}_{20} \mathrm{O}_{6} \mathrm{~N}_{2}\end{array}}_{408 \cdot 2}$

Die Acetylbestimmungen nach Wenzel ergaben:

I. $0 \cdot 3505 \mathrm{~g}$ Substanz verbrauchten $18 \cdot 88 \mathrm{~cm}^{3} 1 / 10 \mathrm{n}$. Kalilauge.

II. $0.4054 \mathrm{~g} \gg, \quad 21.53 \mathrm{~cm}^{3} 1 / 10^{~}$ "

In 100 Teilen:

$\overbrace{\text { Acetyl } \ldots . .23 \cdot 18}^{\text {Gefunden }} \underbrace{22 \cdot 85}_{\text {II }} \quad \underbrace{\begin{array}{c}\text { Berechnet für } \\ \mathrm{C}_{22} \mathrm{H}_{20} \mathrm{O}_{6} \mathrm{~N}_{2}\end{array}}_{21 \cdot 08}$




\section{Einwirkung des Phenylmagnesiumbromids auf 1-Methylisatin.}

Es wurde 1 Mol Methylisatin in die Phenylmagnesiumbromidlösung ( $21 / 2$ Mole) eingetragen. Die Reaktion ist eine ziemlich ruhig verlaufende. Die Aufarbeitung wird in der üblichen Weise vorgenommen. Der Äther wird nach der Zersetzung der Magnesiumverbindung durch verdünnte Schwefelsäure abdestilliert und sodann durch Einleiten von Wasserdampf, Benzol, Diphenyl, Brombenzol usw. abgeblasen. Das harzige Reaktionsprodukt wird abgesaugt und der braune Kuchen in einer Reibschale mit Kalilauge verrieben, um unverändertes Methylisatin zu entfernen. Man saugt neuerlich ab, wäscht mit Wasser gründlich nach und reinigt durch Lösen in warmem Methylalkohol und Fällen der filtrierten methylalkoholischenLösung durch Wasserzusatz. Man erhält so ein gelbes mikrokrystallinisches Pulver, welches zunächst im Vakuum über Schwefelsäure getrocknet wird. Zur Analyse wurde eine Probe in siedendem Ligroin gelöst. Beim Erkalten und beim Abdunsten des Lösungsmittels scheidet sich die Substanz zunächst harzig ab und wird erst allmählich krystallinisch.

I. $0 \cdot 1852 g$ vakuumtrockener Substanz lieferten $0 \cdot 5691 \mathrm{~g} \mathrm{CO}_{2}$ und $0 \cdot 0993 \mathrm{~g}$ $\mathrm{H}_{2} \mathrm{O}$.

II. $0 \cdot 2003 \mathrm{~g}$ Substanz lieferten $0 \cdot 6147 \mathrm{~g} \mathrm{CO}_{2}$ und $0 \cdot 1027 \mathrm{~g} \mathrm{H}_{2} \mathrm{O}$.

III. $0.2870 \mathrm{~g}$ Substanz lieferten $12 \mathrm{~cm}^{3}$ trockenen Stickstoff bei $20^{\circ} \mathrm{C}$, und $742 \mathrm{~mm}$ Barometerstand.

In 100 Teilen:

\begin{tabular}{|c|c|c|c|c|}
\hline & \multicolumn{3}{|c|}{ Gefunden } & \multirow{2}{*}{$\begin{array}{c}\text { Berechnet für } \\
\mathrm{C}_{21} \mathrm{H}_{17} \mathrm{ON}\end{array}$} \\
\hline & I & II & III & \\
\hline$C \ldots \ldots \ldots$ & $83 \cdot 80$ & $83 \cdot 70$ & - & $84 \cdot 22$ \\
\hline H. . & $5 \cdot 99$ & $5 \cdot 73$ & - & $5 \cdot 72$ \\
\hline$N \ldots \ldots \ldots$ & - & - & $4 \cdot 76$ & $4 \cdot 69$ \\
\hline
\end{tabular}

I. $0 \cdot 1022 g$ Substanz erniedrigten den Gefrierpunkt von $14 \cdot 66 g$ Benzol um $0 \cdot 129^{\circ}$.

II. $0 \cdot 1095 \mathrm{~g}$ Substanz erniedrigten den Gefrierpunkt von $15 \cdot 04 g$ Benzol um $0 \cdot 135^{\circ}$. 
794 M. Kohn u. A. Ostersetzer, Kenntnis der 1-Methylisatine.

Daraus berechnetes Molekulargewicht:

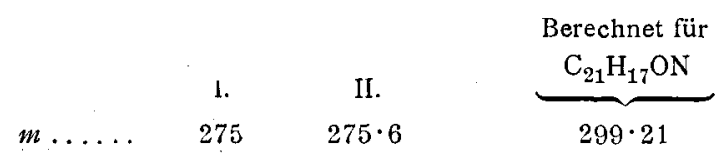

Der Schmelzpunkt liegt um $145^{\circ}$. Das Krystallisationsvermögen ist ein geringes. Das mikroskopische Bild scheint auf monokline tafelförmige Krystalle zu deuten, welche zum Teil unvollkommen ausgebildet sind. Charakteristisch für die Substanz ist die grüne Fluoreszenz der alkoholischen Lösungen.

Die Arbeit wird fortgesetzt. 